\title{
Análise do perfil de segurança de medicamentos fitoterápicos no Brasil: revisão de
}

\section{literatura}

\author{
Analysis of the safety profile of herbal medicines in Brazil: literature review \\ Análisis del perfil de seguridad de los medicamentos a base de hierbas en Brasil: revisión de la
}

literatura

Recebido: 21/09/2021 | Revisado: 01/10/2021 | Aceito: 05/10/2021 | Publicado: 09/10/2021

\author{
Natasha Costa da Rocha Galucio \\ ORCID: https://orcid.org/0000-0003-4923-1478 \\ Escola Superior da Amazônia, Brasil \\ E-mail: natashagalucio@gmail.com \\ Regianne Maciel dos Santos Correa \\ ORCID: https://orcid.org/0000-0002-9837-4304 \\ Escola Superior da Amazônia, Brasil \\ E-mail: regianne83@hotmail.com \\ Daniele de Araújo Moysés \\ ORCID: https://orcid.org/0000-0001-6956-1381 \\ Escola Superior da Amazônia, Brasil \\ E-mail: quimica.dani@gmail.com \\ Paulo Maurício Feliz Paixão \\ ORCID: https://orcid.org/0000-0001-8243-8417 \\ Escola Superior da Amazônia, Brasil \\ E-mail: paulomauriciopaixao@gmail.com \\ Jeferson Rodrigo Souza Pina \\ ORCID: https://orcid.org/0000-0002-9576-8981 \\ Escola Superior da Amazônia, Brasil \\ E-mail: konanquim@gmail.com \\ Gleicy Kelly China Quemel \\ ORCID: https://orcid.org/0000-0003-1280-560X \\ Escola Superior da Amazônia, Brasil \\ E-mail: gkcquemel@gmail.com \\ Valdicley Vieira Vale \\ ORCID: https://orcid.org/0000-0001-6570-4875 \\ Escola Superior da Amazônia, Brasil \\ E-mail: valdicleyvale@gmail.com
}

\begin{abstract}
Resumo
Os medicamentos fitoterápicos têm longa história de uso tradicional no Brasil, entretanto a maioria não tem eficácia e segurança comprovada. O objetivo geral desta pesquisa foi analisar o perfil de segurança de fitoterápicos no Brasil. Este estudo justifica-se pela carência de elucidação acerca da segurança do uso destes produtos, visto que alguns fabricantes de medicamentos fitoterápicos não apresentam os recursos necessários para financiar pesquisas que os respaldem. O corrente estudo foi desenvolvido por meio de pesquisa bibliográfica, do tipo descritiva, utilizando trabalhos dos últimos dez anos, com busca em bases de dados como Lilacs, SciELO e PubMed, a partir das palavraschave: segurança, fitoterápicos e Brasil. Os resultados demonstram a falta de controle de qualidade principalmente microbiológico para fitoterápicos, e afrouxamento das fiscalizações na indústria responsável por sua produção. Com isso, nota-se a necessidade de maiores estudos sobre os fitoterápicos, bem como ampliação das medidas de segurança e fiscalização que os amparam para que a expansão de sua aplicabilidade seja maior, e desta forma, a conduta terapêutica adotada seja eficaz, garantindo menos efeitos colaterais e adversos, bem como preservação de segurança aos usuários.
\end{abstract}

Palavras-chave: Segurança; Fitoterápicos; Brasil.

\begin{abstract}
Herbal medicines have a long history of traditional use in Brazil, however most do not have proven efficacy and safety. The general objective of this research was to analyze the safety profile of herbal medicines in Brazil. This study is justified by the lack of elucidation about the safety of using these products, since some manufacturers of herbal medicines do not have the necessary resources to finance research that support them. The current study was developed through bibliographic research, descriptive, using works from the last ten years, with a search in databases such as Lilacs, SciELO and PubMed, using the keywords: security, herbal medicines and Brazil. The results
\end{abstract}


demonstrate the lack of quality control, mainly microbiological for herbal medicines, and the loosening of inspections in the industry responsible for their production. Thus, there is a need for further studies on herbal medicines, as well as expansion of safety and inspection measures that support them so that the expansion of their applicability is greater, and thus, the therapeutic approach adopted is effective, ensuring less side and adverse effects, as well as preserving user safety.

Keywords: Security; Herbal medicines; Brazil.

\section{Resumen}

Las medicinas a base de hierbas tienen una larga historia de uso tradicional en Brasil, sin embargo, la mayoría no tiene eficacia y seguridad probadas. El objetivo general de esta investigación fue analizar el perfil de seguridad de las medicinas a base de hierbas en Brasil. Este estudio se justifica por la falta de esclarecimiento sobre la seguridad del uso de estos productos, ya que algunos fabricantes de medicamentos a base de hierbas no cuentan con los recursos necesarios para financiar investigaciones que los respalden. El presente estudio se desarrolló a través de una investigación bibliográfica, descriptiva, utilizando trabajos de los últimos diez años, con una búsqueda en bases de datos como Lilacs, SciELO y PubMed, utilizando las palabras clave: seguridad, hierbas medicinales y Brasil. Los resultados demuestran la falta de control de calidad, principalmente microbiológico de los medicamentos herbarios, y la relajación de las inspecciones en la industria responsable de su producción. Por lo tanto, es necesario realizar más estudios sobre los medicamentos herbarios, así como ampliar las medidas de seguridad e inspección que los respalden para que la expansión de su aplicabilidad sea mayor, y así, el abordaje terapéutico adoptado sea efectivo, asegurando menos efectos secundarios y adversos. efectos, además de preservar la seguridad del usuario.

Palabras clave: Seguridad; Hierbas medicinales; Brasil.

\section{Introdução}

Entende-se que os critérios de eficácia e segurança dos medicamentos complementares devem ser os mesmos dos convencionais. Muitos medicamentos complementares, principalmente fitoterápicos, têm uma longa história de uso tradicional. No entanto, a maioria tem eficácia não comprovada pelos padrões atuais, ou seja, ensaios clínicos randomizados bem desenhados e um histórico de uso tradicional não inclui uma avaliação adequada de segurança. A falta de evidências não significa necessariamente que os medicamentos complementares não sejam eficazes, ou seja, inseguros, mas que ainda não foi realizada uma investigação clínica rigorosa e que ainda não foi realizada uma vigilância exaustiva da utilização de medicamentos complementares (Duarte-Almeida, 2021).

Alguns produtos, como certos extratos fitoterápicos padronizados, passaram por extensa investigação clínica, e os ensaios clínicos envolvendo esses medicamentos fitoterápicos foram sujeitos a revisão sistemática/meta-análise, incluindo revisões Cochrane. No entanto, como a composição dos produtos varia entre os fabricantes, as evidências de eficácia (e segurança) devem ser consideradas como específicas do extrato. No máximo, as evidências devem ser extrapoladas apenas para preparações da mesma erva com um perfil de constituintes muito semelhantes (Angonesi \& Sevalho, 2010). Por exemplo, a maioria dos ensaios clínicos de Ginkgo (Ginkgo biloba) testou os extratos de folhas de Ginkgo padronizados EGb-761 e LI1370. Não se deve presumir que os resultados desses estudos se aplicam a outros extratos de folha de Ginkgo, que podem ter um perfil de constituintes diferente, ou a outras preparações da folha de Ginkgo, como tinturas e chás. No entanto, muitas revisões sistemáticas e meta-análises de ensaios clínicos de medicamentos fitoterápicos ignoram detalhes importantes dos produtos testados, como o tipo de extrato e a formulação (Silva et al., 2012).

Ao contrário de certos medicamentos, sobre os fitoterápicos, há escassez de pesquisas científicas sobre os efeitos de alguns tipos de medicamentos complementares, como os florais de Bach. Está além do escopo desta visão geral resumir todas as evidências clínicas relacionadas aos medicamentos complementares (ANVISA, 2011). Logo o problema deste estudo gira em torno do seguinte questionamento: Qual o perfil de análise de segurança de fitoterápicos no Brasil?

O objetivo geral desta pesquisa foi analisar o perfil de segurança de fitoterápicos no Brasil. O estudo justifica-se na medida em que as empresas farmacêuticas do país são grandes patrocinadores da pesquisa médica, mas alguns fabricantes de medicamentos complementares não têm os recursos necessários para realizar ou financiar pesquisas envolvendo seus produtos. 
Além disso, há pouco incentivo para a realização de pesquisas porque os medicamentos complementares, como produtos naturais não podem ser patenteados, portanto, os fabricantes não têm um período de proteção no qual possam recuperar o retorno financeiro dos investimentos em pesquisa e desenvolvimento (Sharma et al., 2010).

Esta pesquisa centralizou-se nos medicamentos fitoterápicos, uma vez que estão entre os "medicamentos complementares" mais usados no Brasil e, de uma perspectiva biomédica, são provavelmente os de maior potencial em termos de risco. No caso dos medicamentos fitoterápicos, geralmente, faltam dados sobre: constituintes ativos, metabólitos, farmacocinética, farmacologia, toxicologia, efeitos adversos e suas frequências, efeitos do uso de longo prazo, interação drogaerva, interações com comida e o álcool, uso em grupos específicos de pacientes (crianças, idosos, indivíduos com doença renal ou hepática, efeitos de gênero, indivíduos com um perfil genético diferente), contraindicações e advertências, e o uso na gravidez e lactação. Algumas questões importantes de segurança que surgiram com medicamentos fitoterápicos específicos foram discutidas (Vieira et al., 2010). No entanto, está além do escopo desta pesquisa revisar as informações sobre os aspectos de segurança de todos os medicamentos fitoterápicos.

\subsection{O Brasil e a Fitoterapia}

O Brasil possui grande potencial para o desenvolvimento de fitoterapias, pois detém a maior diversidade vegetal e social do mundo. $\mathrm{O}$ uso de plantas medicinais foi vinculado aos saberes tradicionais populares e à projeção tecnológica, a fim de validar cientificamente tais conhecimentos. A utilização dessas plantas significa produzir e reproduzir campos e práticas de saberes oriundos de diferentes culturas e da organização social e produtiva em comunidades tradicionais (Fontenele et al., 2013).

O uso de espécies de plantas para tratar e curar doenças foi perpetuado ao longo da história da civilização. Quanto à importância dada pelos homens primitivos às plantas, os feiticeiros eram considerados intermediários de Deus com os homens, eles tinham a tarefa de curar doenças. Há práticas que articulam magia, religião e práticas empíricas de saúde, como o uso de plantas medicinais, que eram vistas como recurso terapêutico em muitas comunidades e etnias. Observações populares e investigações científicas sobre o uso e a eficácia das plantas medicinais em todo o mundo destacam a prática de consumo de produtos fitoterápicos e valida as informações coletadas ao longo dos séculos (Thiago \& Tesser, 2011).

A Etnobotânica e a Etnofarmacologia são consideradas ferramentas importantes para resgatar os conhecimentos tradicionais das sociedades humanas passadas e presentes, e suas interações ecológicas, genéticas, evolutivas, simbólicas e culturais com as plantas, bem como com o desenvolvimento do conhecimento científico e tecnológico sobre a sustentabilidade uso de recursos naturais (Araújo et al., 2014).

O Brasil tem enfrentado muitos avanços nas últimas décadas no que se refere à regulamentação da fitoterapia e sua aceitação como estratégia terapêutica disponível no Sistema Único de Saúde (SUS). A formulação e implementação de políticas públicas de apoio à ampliação do conhecimento sobre as plantas medicinais tradicionais e científicas e sua utilização na atenção básica pelo Sistema Único de Saúde representa o marco regulatório e o incentivo à pesquisa em plantas medicinais e fitoterapia (Varela, 2014).

\subsection{Plantas medicinais e o Conhecimento tradicional}

De acordo com a Organização Mundial da Saúde (OMS) grande parte da população mundial confia nos métodos tradicionais relacionados à saúde diária. Aproximadamente $80 \%$ da população, principalmente nos países em desenvolvimento, dependem de derivados de plantas medicinais para sua própria saúde. Aproximadamente $25 \%$ de todas as prescrições de medicamentos são formulações à base de substâncias derivadas de plantas ou análogos sintéticos ou de seus derivados (Oliveira et al., 2012). 
As potencialidades desses medicamentos são amplamente valorizadas pela população tradicional independentemente dos aspectos culturais e dos benefícios compartilhados decorrentes de tais conhecimentos. Inúmeros processos marcaram a arte de curar por meio das plantas medicinais. Porém, é difícil delimitá-los com precisão, visto que, por muito tempo, foram associados a práticas mágicas, místicas e ritualísticas, fato que lhes conferiu importância vital na medicina tradicional. As mulheres ficavam encarregadas de extrair os princípios ativos das plantas para serem usados na cura de doenças nas tribos brasileiras (Rosa et al., 2011).

À medida que os indivíduos se tornam capazes de atender às suas necessidades de sobrevivência, eles começam a estabelecer papéis sociais específicos para os membros da comunidade. O papel principal cabia ao "curandeiro", que devia zelosamente desenvolver e guardar um repertório de substâncias secretas e transmitir seletivamente esse conhecimento a iniciantes bem preparados (Ribeiro \& Guimarães, 2013).

Uma série de influências culturais, como a dos colonizadores europeus, indígenas e africanos, levou ao conhecimento popular. Essas influências eram geralmente desenvolvidas por grupos culturais que vivem intimamente em contato com a natureza e a observam de perto no dia a dia. Esses grupos exploram o potencial e mantêm vivo o conhecimento herdado devido à experimentação sistemática e constante. Eles acreditam que essas plantas são uma alternativa viável para o tratamento de doenças ou para a manutenção da saúde (Bruning et al., 2012).

O Brasil era essencialmente rural até meados do século XX e apresentava amplo uso de flora medicinal, tanto nativa quanto introduzida. Porém, em muitas de suas localidades, onde a modernização havia sido introduzida com mais força, a convivência com a flora medicinal deixou de existir, devido às mudanças na aceitação dos valores tradicionais por parte dos jovens que passaram a enxergar o saber popular como antiquado e depreciativo. $O$ início da industrialização e a crescente urbanização colocaram os saberes tradicionais em segundo plano devido às pressões econômicas e culturais externas. Também ocorreram mudanças na vida, valores e conceitos lógicos durante o processo de socialização. Tais mudanças estiveram associadas às transformações sociais e, consequentemente, desvalorizaram essas práticas tradicionais. O conhecimento tradicional foi se perdendo com o tempo, uma vez que os indivíduos não deixaram nenhum registro escrito deles. Os novos hábitos sociais sempre priorizam as informações estrangeiras e facilitam o acesso aos serviços da medicina moderna. Assim, novos hábitos sociais levam ao deslocamento dos moradores locais de seus ambientes naturais para as áreas urbanas (Dutra, 2009).

Durante a segunda metade do século $\mathrm{XX}$, as questões ambientais ganharam espaço em painéis internacionais. No entanto, um forte processo de insurgência envolvendo outros atores lutou pela "defesa da natureza" ou "desenvolvimento sustentável"; veio junto com a popularização do movimento ambientalista. Os esforços políticos empreendidos ainda não respondem de forma satisfatória aos conflitos atuais do cotidiano. Como o modelo capitalista e todas as formas de materialização têm dominado quase todo o globo, as populações de vida tradicional (indígenas, quilombolas, comunidades rurais e comunidades periféricas dos centros urbanos) foram deixadas à margem do desenvolvimento. Além disso, por muito tempo, as representações da medicina tradicional foram consideradas objetos exóticos, sem coerência e eficiência. Apresentaram sociedades e culturas atrasadas condenadas ao desaparecimento em função da implantação e disseminação da Medicina Ocidental, antes das diferentes manifestações dessas práticas realizadas por curandeiros e "especialistas" em plantas (Cantarelli, 2012).

Por viverem em contato com o meio ambiente, agricultores e comunidades tradicionais brasileiras incorporaram elementos da paisagem florestal em sua rotina produtiva como estratégia de sobrevivência para obter diferentes recursos para o consumo próprio da família, atender às necessidades de sua atividade produtiva e ter sazonalidade ou ocasionalmente um segundo rendimento ao longo do ano. $\mathrm{O}$ uso regional dos recursos nativos está fortemente associado às comunidades locais, pequenas propriedades, caiçaras, quilombolas e comunidades indígenas. Eles usam esses recursos para fins energéticos (lenha), 
para o consumo de frutas e plantas da estação, para fins ornamentais e medicinais. Devido à disponibilidade de matéria-prima, as plantas medicinais costumam ser cultivados em jardins, quintais ou colhidas na mata, como os ancestrais faziam. A precariedade do atendimento médico convencional fez das plantas o único recurso de tratamento de doenças disponível na maioria dos casos (Santos et al., 2014).

$\mathrm{O}$ conhecimento nas comunidades tradicionais muitas vezes parece ligado à sua praticidade. Assim, o conhecimento interconecta experiências e interfere de forma real no ambiente da comunidade; na maioria das vezes é fonte de surgimento de novos conhecimentos. A consciência geralmente tem algum propósito e leva a resultados práticos. Não há discriminação entre conhecimento teórico e prático nessas comunidades, pois ambos são adquiridos ao mesmo tempo. As crianças participam das tarefas diárias, absorvem gradativamente as explicações verbais e codificam-nas por meio de seus próprios fazeres. Essas comunidades, na maioria das vezes, são grupos familiares que detêm seus próprios conhecimentos e os transferem de geração em geração, ou seja, transferem suas características, cultura e a forma como lidam com a saúde dentro do próprio grupo. Esse tipo de contato familiar é muito comum em comunidades rurais onde a agricultura familiar é efetiva e o contato diário entre gerações é valorizado. Não transferem apenas os aspectos relacionados ao sistema de produção sustentável, mas também aqueles associados às crenças, valores e ao meio cultural (Varela, 2014).

A consciência sobre a forma como as diferentes sociedades lidam com o meio envolvente desempenha papel fundamental no acesso aos saberes tradicionais socializados, na medida em que tal consciência é registrada e sistematizada como tentativa de resgate do saber popular. Pesquisas sobre o assunto têm sido relevantes e vêm somar-se à reflexão sobre a relação humana com o meio ambiente em um determinado tempo e lugar. Tais levantamentos podem subsidiar planos de manejo florestal fim de alcançar a conservação da biodiversidade e tornar esse tipo de conhecimento acessível às gerações futuras e para estudos posteriores (Cantarelli, 2012).

Todos os elementos submetidos ao estudo são importantes para esclarecer conceitos e práticas relacionadas à saúde e à cura de doenças em uma determinada comunidade. A Etnobotânica e a Etnofarmacologia das plantas medicinais tradicionais são importantes ferramentas para garantir o resgate do "conhecimento", levando em consideração sua aplicabilidade científica tradicional. Esses dois campos científicos buscam uma lista de plantas para auxiliar a pesquisa de substâncias bioativas e esclarecer os elementos constitutivos e característicos (materiais ou simbólicos) e conceitos das práticas terapêuticas locais (Thiago \& Tesser, 2011).

\subsection{A importância da fitoterapia para o SUS - Sistema Único de Saúde}

Historicamente, as plantas medicinais são importantes e auxiliam na descoberta de novos medicamentos. A maior parte das contribuições médicas vem do reino vegetal. O termo "fitoterapia" é dado a tratamentos que utilizam plantas ou seus derivados como componente ativo. Esses tratamentos resultam do conhecimento popular e utilizam plantas tradicionalmente chamadas de "plantas medicinais" (Fontenele et al., 2013).

O uso de fitoterápicos com finalidade profilática, curativa, paliativa ou diagnóstica tornou-se oficialmente reconhecido pela Organização Mundial da Saúde (OMS) em 1978, quando recomendou a divulgação mundial do conhecimento necessário para seu uso. Ao considerar as plantas medicinais como importantes instrumentos da Assistência Farmacêutica, é possível afirmar que muitos editais e resoluções da OMS expressam o posicionamento da organização quanto à necessidade de valorizar o uso desses medicamentos no âmbito sanitário. Recomenda também que as políticas nacionais de regulação, no que se refere aos produtos derivados das práticas tradicionais, sigam os conceitos da Medicina Tradicional (MT) e da Medicina Complementar/Alternativa (MAC) entre outros (Rosa et al., 2011).

Produtos e serviços oferecidos pelo Sistema Único de Saúde (SUS), como plantas medicinais e programas de fitoterapia, seguem procedimentos distintos, uma vez que as espécies de plantas medicinais disponíveis estão distribuídas (em 
todas as regiões brasileiras) de acordo com diferentes biomas. Estudos sobre programas e ações de fitoterapia mostram que a inclusão da fitoterapia e das plantas medicinais nas unidades básicas de saúde tem facilitado o acesso a outras possibilidades terapêuticas, além das drogas sintéticas. Fortalece a implementação de políticas públicas, o desenvolvimento local e o resgate de populações tradicionais baseadas no conhecimento. Tal inclusão estimula os profissionais de saúde a organizarem atividades de educação sanitária e ambiental e a realizarem ações intersetoriais (parcerias agrícolas, educacionais e ambientais) (Santos et al, 2014).

Atualmente, os principais instrumentos utilizados para orientar o desenvolvimento de ações/programas envolvendo plantas medicinais e fitoterapia são: a Política Nacional de Práticas Integrativas e Complementares do Sistema Único de Saúde (PNPIC-SUS - Política Nacional de Práticas integrativas e Complementares do Sistema Único de Saúde) e suas diretrizes sobre Plantas Medicinais e Fitoterapia no Sistema Único de Saúde (SUS) e a Política Nacional de Plantas Medicinais e Fitoterápicos (PNPMF - Política Nacional de Plantas Medicinais e Fitoterápicos). Essas ações/programas contemplam o desenvolvimento e adaptação de marcos regulatórios e fomentam a pesquisa com plantas medicinais. Eles priorizam a biodiversidade no país e o desenvolvimento de tecnologias e inovações nas diferentes etapas da cadeia produtiva (Bruning et al., 2012).

O PNPMF incentiva pesquisas envolvendo plantas exóticas ou nativas adaptadas incluídas na Lista Nacional de Fitoterápicos do Brasil (RENAFITO - Relação Nacional de Plantas Medicinais e Fitoterápicos). Esta lista é subsidiada por estudos e pesquisas sobre desenvolvimento e inovação de produtos, principalmente no setor agroindustrial. Os critérios de inclusão do RENAFITO são: i) ser uma espécie da flora brasileira fora de risco de extinção; ii) estar presente em diversos biomas brasileiros; iii) para evidenciar segurança e eficácia; iv) ser registrado na Agência Nacional de Vigilância Sanitária ANVISA; v) ser um fitoterápico utilizado na atenção primária; vi) ser produzido com plantas exóticas nativas ou adaptadas (Araújo et al., 2014).

O Ministério da Saúde (MS) publicou em fevereiro de 2009 a Relação Nacional de Plantas Medicinais de Interesse ao SUS (RENISUS), com o objetivo de fortalecer a pesquisa sobre plantas medicinais nativas e disponibilizar essas informações. A lista é composta por 71 espécies de plantas pesquisadas; são priorizados os investimentos neles e seu uso seguro e eficaz nas diferentes formas de apresentação: material vegetal in natura, planta desidratada e medicamentos manipulados e manufaturados em farmácia. A comprovação de segurança e eficácia é realizada de acordo com o uso tradicional listado na literatura ou em estudos científicos sobre essas espécies de plantas (Ribeiro \& Guimaraes, 2013).

Diferentemente do que se pode pensar, a Fitoterapia é uma prática complementar à saúde, embora seja uma das mais conhecidas e utilizadas nos serviços públicos brasileiros. O crescente número de profissionais de saúde dispostos a se formar nessa área foi percebido em alguns municípios brasileiros, uma vez que todos aspiram a melhorar a cada dia seu trabalho na área da saúde e adquire novas ferramentas. A expansão da Estratégia Saúde da Família (ESF) - movimento estruturante da atenção básica no SUS (Sistema Único de Saúde) - abriu importante espaço de trabalho para a Fitoterapia (Cantarelli, 2012).

O programa desenvolveu princípios a serem aplicados a esse nível de atenção. As particularidades da fitoterapia envolvem a interação entre saberes, parcerias em saúde e ações de promoção e prevenção na atenção básica. As ações fitoterápicas fortalecem o vínculo entre usuários, comunidade e equipes da ESF, bem como entre a participação popular, a autonomia do usuário e os sistemas de saúde. Portanto, a expansão da cobertura da Atenção Básica em diferentes regiões e biomas indica o potencial para o desenvolvimento de plantas medicinais e a adoção de ações fitoterápicas nos serviços públicos de saúde onde a fitoterapia pode ser oferecida (Dutra, 2009).

Portanto, o acesso da população às práticas e benefícios fitoterápicos na atenção básica torna-se um terreno fértil para o desenvolvimento de suas potencialidades em um campo multiprofissional, portanto, também abre uma ampla gama de possibilidades e vantagens. A proximidade entre os profissionais de saúde e a comunidade é uma tentativa de horizontalizar a 
relação entre eles e reforça o papel dos serviços de atenção básica - o primeiro contato dos usuários com o SUS. A expansão da oferta de saúde remete ao princípio da integralidade da saúde (Thiago \& Tesser, 2011).

Esse campo científico atuante na fitoterapia, tornou-se de extrema importância quando muitos dos estudos aqui citados relatam que o conhecimento tradicional está se perdendo ao longo do tempo, seja pela morte de indivíduos que não deixaram registros escritos, seja pela introdução de novos hábitos nas sociedades modernas. Estudos sobre programas e ações fitoterápicos mostram que a inclusão de plantas fitoterápicas e medicinais na atenção básica melhorou o acesso a outras possibilidades terapêuticas, além das drogas sintéticas. Esses estudos têm fortalecido a implementação de políticas públicas e o desenvolvimento local. Também resgataram os saberes tradicionais de antigas comunidades, ajudando assim a divulgar a investigação científica e a desenvolver uma visão crítica dos profissionais e da população sobre o uso adequado das plantas medicinais e fitoterápicos (Oliveira et al., 2012). Pelo exposto, é possível perceber a importância de se analisar o perfil de segurança de fitoterápicos no Brasil.

\section{Metodologia}

Trata-se de uma revisão de literatura (Pereira et al., 2018), sobre o perfil de segurança de medicamentos fitoterápicos no Brasil. Para esta pesquisa, as buscas foram realizadas utilizadas em bases de dados como Lilacs, Scielo e PubMed, a partir das seguintes palavras-chave: Segurança; Fitoterápicos; Brasil. Foram utilizados filtros para estabelecer a seleção de artigos dos últimos dez anos (2011-2021), como demonstrado no Quadro 1. Foram considerados como critérios de inclusão artigos completos de literatura científica em língua portuguesa, e foram excluídos os que não abordam sobre a temática e estejam fora do recorte temporal estipulado. Para a revisão de literatura, os artigos selecionados com duplicidade, foram removidos e incluídos utilizando os mesmos critérios de elegibilidade e triagem. Os trabalhos foram selecionados com o auxílio do programa Rayyan QCRI.

Quadro 1 - Estratégia de busca utilizada em banco de dados.

\begin{tabular}{|c|c|c|c|c|}
\hline Estratégia & Palavras-chaves & Base de dados & Filtro & $\mathbf{N}^{\mathbf{0}}$ artigos \\
\hline 1 & Segurança; Fitoterápicos; Brasil & Lilacs & $2011-2021$ & 37 \\
\hline 2 & Segurança; Fitoterápicos; Brasil & SciELO & $2011-2021$ & 3 \\
\hline 3 & Segurança; Fitoterápicos; Brasil & PubMed & $2011-2021$ & 0 \\
\hline
\end{tabular}

Fonte: Autores.

\section{Resultados}

Este estudo identificou 40 artigos em três diferentes bases de dados, dos quais 5 eram duplicados, e consequentemente excluídos. Na fase de triagem, foram excluídos 31 artigos (Quadro 2), pois: 
Quadro 2 - Relação de artigos excluídos da pesquisa.

\begin{tabular}{|c|l|}
\hline 1 & Tratava-se de modelo de melhoria, o qual avaliava a aplicabilidade de testes rápidos. \\
\hline 11 & Tratavam-se de estratégias nutricionais. \\
\hline 1 & Tratava-se da relação de cana-de-açúcar e internações hospitalares. \\
\hline 3 & Tratavam-se de ações de Vigilância em Saúde do Trabalhador. \\
\hline 2 & Tratavam-se da utilização de Agrotóxicos. \\
\hline 7 & Tratavam-se de pesquisas de Saúde Pública, considerando seus aspectos éticos, gerenciais e de segurança. \\
\hline 1 & Tratava-se de cobertura por plano de saúde. \\
\hline 1 & Tratava-se do cuidado hospitalar. \\
\hline 2 & Tratavam-se de decisão judicial acerca do uso de medicamentos. \\
\hline 1 & Tratava-se de toxoplasmose congênita. \\
\hline 1 & Tratava-se do levantamento bibliográfico acerca de medicamentos fitoterápicos no hall do Ministério da Saúde \\
\hline
\end{tabular}

Fonte: Autores.

Assim, apenas 4 artigos foram incluídos para síntese qualitativa, dentre estes (quadro 3). Uma publicação levantada nesta revisão analisou documentos do Ministério da Saúde sobre a construção da política para a implantação da Fitoterapia no SUS, além de revisar artigos sobre aspectos da Fitoterapia em serviços de saúde. O autor considera que os conhecimentos sobre a Fitoterapia estão cada vez mais profundos, pois a implementação deste assunto em ambientes acadêmicos tem ampliado sua disseminação. Entretanto, o assunto ainda é cercado por muito descrédito e falta de embasamento científico, pois mesmo com todo o crescimento, a prática ainda não é utilizada com tanta frequência na atenção primária (Figueiredo et al., 2014).

Uma dissertação de mestrado avaliou artigos sobre aspectos da Vigilância Sanitária de Medicamentos relacionadas a produção, distribuição, transporte, aonde os medicamentos de maior interesse de para o estudo foram os fitoterápicos (Silva, 2013). O estudo obteve resultados que demonstram a falta de ações normativas e fiscalização que assegurem a eficácia e segurança destes medicamentos.

Outro estudo analisou os requisitos para a comprovação da eficácia e segurança de medicamentos fitoterápicos e drogas vegetais industrializados, bem como a qualidade dos ensaios clínicos realizados. Seus resultados demonstram que para o processo de regulamentação e registro de medicamentos fitoterápicos não são exigidas as mesmas especificações técnicas quando comparado a medicamentos puramente químicos. Desta forma, medicamentos fitoterápicos possuem maior facilidade e menos processos burocráticos para seu registro, com menor apresentação de evidências científicas que embasem a utilização terapêutica (Teixeira, 2013).

Santos et al. (2014) observaram que o Brasil tem instaurado políticas públicas que visam o avanço tecnológico a assistência de saúde básica eficaz, e que seja capaz de abranger maior número de pessoas com menor relação de dependência aos insumos provenientes da indústria farmacêutica. E muito embora a implantação de fitoterápicos na atenção primária a saúde seja cada vez maior, os estudos acerca desta temática ainda são escassos. Desta forma, os autores enfatizam a 
necessidade do maior número de pesquisas sobre a segurança de práticas fitoterápicas no SUS, para que sua implantação e eficácia sejam ampliadas (Quadro 3).

Quadro 3 - Resultados extraídos de cada estudo revisado.

\begin{tabular}{|l|l|}
\hline Artigos & Resultados \\
\hline $\begin{array}{l}\text { A Política Nacional de Plantas Medicinais e } \\
\text { 2014). }\end{array}$ & $\begin{array}{l}\text { Os autores consideram que os conhecimentos sobre a } \\
\text { Fitoterapia estão cada vez mais profundos, pois a } \\
\text { implementação deste assunto em ambientes acadêmicos têm } \\
\text { ampliado sua disseminação. Entretanto, o assunto ainda é } \\
\text { cercado por muito descrédito e falta de embasamento } \\
\text { científico, pois mesmo com todo o crescimento, a prática ainda } \\
\text { não é utilizada com tanta frequência na atenção primária. }\end{array}$ \\
\hline $\begin{array}{l}\text { Aspectos da Produção Científica da Vigilância } \\
\text { Sanitária de Medicamentos (Silva, 2013). }\end{array}$ & $\begin{array}{l}\text { O estudo obteve resultados que demonstram a falta de ações } \\
\text { normativas e fiscalização que assegurem a eficácia e segurança } \\
\text { destes medicamentos. }\end{array}$ \\
\hline $\begin{array}{l}\text { Medicamentos Fitoterápicos e Drogas Vegetais } \\
\text { Industrializados e Oficializadospelo Ministério da } \\
\text { Saúde no Brasil: Regulamentação Sanitária, } \\
\text { Abrangência Equalidade dos Estudos Pré-Clínicos e } \\
\text { Clínicos (Teixeira, 2013). }\end{array}$ & $\begin{array}{l}\text { Os resultados apontam para critérios menos rígidos quanto a } \\
\text { regulamentação do processo de registro de fitoterápicos quando } \\
\text { comparados aos medicamentos quimicos. }\end{array}$ \\
$\begin{array}{l}\text { Análise Sobre a Fitoterapia Como Prática Integrativa } \\
\text { no Sistema Único de Saúde (Santos et al., 2014). }\end{array}$ & $\begin{array}{l}\text { Os autores reconhecem o recente crescimento acerca de } \\
\text { políticas públicas para maior implantação de fitoterápicos na } \\
\text { atenção primária, porém, estudos sobre este viés ainda são } \\
\text { escassos, não garantindo a devida segurança e eficácia. }\end{array}$ \\
\hline
\end{tabular}

Fonte: Autores.

\section{Discussão}

A prática fitoterápica vem apresentando amplo crescimento na contemporaneidade. Com isso, esta prática de medicina alternativa tem apresentado crescimento, haja vista seu baixo custo e real eficácia de plantas medicinais, bem como a crença que medicamentos químicos possuem mais fatores de agressão ao organismo humano (Silva, 2013).

$\mathrm{O}$ corrente estudo apresenta resultados de autores que consideram inúmeros benéficos da fitoterapia para o Sistema Público de Saúde, haja vista a acessibilidade das plantas e ervas no Brasil, bem como apresentarem fácil utilização caseira e serem populares ao conhecimento empírico no emprego de tratamento de diversas doenças (Santos et al., 2014).

No entanto, estudo como o de Teixeira (2013), demonstrado que as exigências burocráticas que regulam a inserção de medicamentos fitoterápicos no mercado são mais brandas, quando comparadas as exigências para regulação de medicamentos sintéticos. Esta afirmação é em decorrência das espécies vegetais que constam na Lista de Medicamentos Fitoterápicos de Registro Simplificado (LMFRS), as quais não são passam por avaliação científica massiva para serem incluídos na lista.

A Vigilância Sanitária de Medicamentos já afirmou ter encontrado resíduos sólidos de contaminação microbiana em medicamentos fitoterápicos, e por isso, foram sugeridas algumas exigências de especificação para estes produtos (Bruning et al., 2012). 
Em consonância, os resultados obtidos por esta pesquisa demonstraram ínfimo nível qualitativo para fitoterápicos, onde já foram encontrados alguns fungos produtores de micotoxinas, além de Aspergillus e Penicillium. Desta forma, nota-se a importância de maior fiscalização ao controle de qualidade microbiológico para a fitoterapia, afim de garantir segurança aos que utilizam da medicação (Santos et al., 2014).

A utilização de plantas medicinais para fins terapêuticos não pode isentar os fabricantes de adequada avaliação científica, pois as mesmas possuem efeitos adversos, apresentam interações com medicamentos sintéticos (Silveira et al., 2008). Leite et al. (2011) ressaltam que, um dos mais valiosos princípios éticos que deve ser levado em consideração, é o qual afirma que o risco não deve ser maior que o benefício oriundo da utilização de uma medicação.

Neste sentido, (Carvalho et al., 2021), sugere mais pesquisas que justifiquem o uso de plantas medicinais na prevenção e tratamento de diversas doenças, destacando o papel da atenção farmacêutica que reúnam informações úteis aos profissionais de saúde para orientar o estudo, sensibilizar, levantar discussões e contribuir para promoção da utilização segura e racional das plantas medicinais.

Quanto sua aplicabilidade em serviços de saúde, Silva (2013), enfatiza a necessidade dos profissionais de saúde receberem treinamentos que os capacitem a prescrição adequada, e garantem a implementação da Fitoterapia no SUS, haja vista que muitas vezes, existem alguns preconceitos sobre suas interações, e a real eficácia durante a condução da terapêutica. O fato supracitado corrobora com a perpetuação do equívoco sobre medicamentos químicos, onde alguns profissionais afirmam com segurança que possuem maior garantia de qualidade, dado o ínfimo número de estudos sobre a temática (DuarteAlmeida, 2021).

Assim, os fitoterápicos necessitam impreterivelmente passar por todas as fases de ensaios clínicos que qualquer outro produto é submetido, pois somente desta maneira é que sua utilização terá respaldo científico, e a devida segurança acerca das interações farmacodinâmicas e cinéticas que o envolvem (Figueiredo et al., 2014).

Por fim, Figueiredo et al. (2014) afirmam que a escassez de evidências científicas que comprovem a eficácia desses medicamentos podem ser um fator que comprometa sua segurança. Desta forma, o profissional responsável pela prescrição deve manter-se atento a estes aspectos, do mesmo modo que o farmacêutico precisa estar atento a sua dispensação, afim de garantir a segurança do paciente em questão.

\section{Considerações Finais}

A utilização de fitoterápicos apresenta múltiplos benefícios, dentre eles seu baixo custo e fácil disponibilidade, bem como conhecimento empírico que já fora popularmente disseminado, no entanto, é necessário maior compreensão sobre o perfil de segurança destes medicamentos. Dado isso, o presente estudo teve como objetivo elucidar aspectos sobre a farmacovigilância de medicamentos fitoterápicos, e identificar a importância da atenção farmacêutica na prescrição de fitoterápicos.

Desta forma, os resultados obtidos demonstram a necessidade de maior controle de qualidade sobre os aspectos de segurança destes medicamentos, bem como ampliação sobre a fiscalização de controle microbiológico, afim de garantir maior segurança e eficácia em sua utilização. Mesmo que estudos sobre a temática venham crescendo, é de fundamental importância que os profissionais de saúde sejam treinados adequadamente para sua correta prescrição, bem como tenham conhecimento sobre os principais pontos de divergência em relação aos medicamentos sintéticos.

Com isso, nota-se a necessidade de maiores estudos sobre os fitoterápicos, bem como ampliação das medidas de segurança e fiscalização que o amparam, para que a expansão de sua aplicabilidade seja maior, e desta forma, a conduta 
terapêutica adotada seja eficaz, garantindo menos efeitos colaterais e adversos, bem como preservação de segurança aos usuários.

\section{Referências}

Angonesi, D., \& Sevalho, G. (2010). Atenção Farmacêutica: fundamentação conceitual e crítica para um modelo brasileiro. Ciência \& Saúde Coletiva, 15(3), 3603-3614.

ANVISA. Agencia Nacional de Vigilância Sanitária (2011). Resolução da diretoria colegiada - RDC nº 24 , de 14 de junho de 2011.

Araujo, W. R. M., Silva, R. V., Barros, C. da S., \& Amaral, F. M. M. do. (2014). Inserção da fitoterapia em unidades de saúde da família de São Luís, Maranhão: realidade, desafios e estratégias. Revista Brasileira De Medicina De Família E Comunidade, 9(32), $258-263$.

Bruning, M. C. R., Mosegui, G. B. G., \& Vianna, C. M. M. (2012). A utilização da fitoterapia e de plantas medicinais em unidades básicas de saúde nos municípios de Cascavel e Foz do Iguaçu - Paraná: a visão dos profissionais de saúde. Ciência \& Saúde Coletiva, 17(10), 2675-2685.

Cantarelli, AP. (2012). Estudo da utilização de plantas medicinais pelos usuários do SUS e das práticas dos profissionais de saúde de doutor Maurício Cardoso em relação à fitoterapia. Monografia, Curso de especialização em Gestão em Saúde/ UAB. Universidade Federal do Rio Grande do Sul. Três Passos, Brasil.

Carvalho, L. O. L. de., Reis, T. S. dos, Quemel, G. K. C., Moysés, D. de A. ., Galucio, N. C. da R. ., \& Correa, R. M. dos S. (2021). Pharmaceutical care in the use of medicinal plants with anti-hypertensive action in the elderly. Research, Society and Development, 10(9), e18010917793.

Duarte-Almeida, J. M., Tabach, R., Kato, E. T. M., Carlini, E. A., Negri, G., Sousa, B. J. M., Carvalho, A. C. B., \& Soares, J. (2021). Boletim Planfavi Sistema de Farmacovigilância em Plantas Medicinais.

Dutra, M. G. (2009). Plantas medicinais, fitoterápicos e saúde pública: um diagnóstico situacional em Anápolis, Goiás. Dissertação de mestrado, Programa de pós-graduação em Sociedade, Tecnologia e Meio Ambiente - Centro Universitário de Anápolis - UniEvangélica, Brasil.

Figueiredo, C. A. D., Gurgel, I. G. D., \& Gurgel Junior, G. D. (2014). A Política Nacional de Plantas Medicinais e Fitoterápicos: construção, perspectivas e desafios. Physis: Revista de Saúde Coletiva, 24(2), 381-400.

Fontenele, R. P., Sousa, D. M. P., Carvalho, A. L. M., \& Oliveira, F. A. (2013). Fitoterapia na Atenção Básica: olhares dos gestores e profissionais da Estratégia Saúde da Família de Teresina (PI). Brasil. Ciência \& Saúde Coletiva, 18(8), 2385-2394.

Leite, S. R., Amorim, M. M., Sereno, P. F., Leite, T. N., Ferreira, J. A., \& Ximenes, R. A. (2011). Randomized clinical trial comparing the efficacy of the vaginal use of metronidazole with a Brazilian pepper tree (Schinus) extract for the treatment of bacterial vaginosis. Brazilian journal of medical and biological research = Revista brasileira de pesquisas medicas e biologicas, 44(3), 245-252.

Oliveira, S. G. D., Moura, F. R. R., Demarco, F. F., Nascente, O. S., Pino, F. A. B., Lund, R.G. (2012). An ethnomedicinal survey on phytotherapy with professionals and patients from Basic Care Units in the Brazilian Unified Health System. J Ethnopharmacol 2012; 140(2):428-437.

Pereira A. S. et al. (2018). Metodologia da pesquisa científica. [free e-book]. Santa Maria/RS. Ed. UAB/NTE/UFSM.

Ribeiro, K. S., \& Guimarães, A. L. A. (2013). O uso de medicamentos à base de plantas medicinais por médicos do SUS no município de Teresópolis/RJ. Revista Agrogeoambiental, 1(1), 61-65.

Rosa, C., Câmara, S. G., \& Béria, J. U. (2011). Representações e intenção de uso da fitoterapia na atenção básica à saúde. Ciência \& Saúde Coletiva, 16(1), $311-318$.

Santos, R. L., Guimaraes, G. P., Nobre, M. S. C., \& Portela, A. S. (2014). Análise sobre a fitoterapia como prática integrativa no Sistema Único de Saúde. Revista Brasileira de Plantas Medicinais, 13(4), 486-491.

Sharma, A. K., Kumar, R., Mishra, A., \& Gupta, R. (2010). Problems associated with clinical trials of Ayurvedic medicines. Revista Brasileira de Farmacognosia, 20(2), 276-281.

Silva, A. I. (2013). Aspectos da produção científica da vigilância sanitária de medicamentos. Dissertação de Mestrado, Faculdade de Saúde Pública, Universidade de São Paulo, São Paulo, Brasil.

Silva, E. R. B., Bandeira, V. A. C., \& Oliveira, K. R. (2012). Avaliação das prescrições dispensadas em uma farmácia comunitária no município de São Luiz Gonzaga - RS. Revista de Ciências Farmacêuticas Básica e Aplicada, 12(2), 275-281.

Silveira, P. F., Bandeira, M. A. M., \& Arrais, P. S. D. (2008). Pharmacovigilance and adverse reactions to the medicinal plants and herbal drugs: a reality. Revista Brasileira de Farmacognosia, 18(4), 618-626.

Teixeira, S. S. T. C. (2013). Medicamentos fitoterápicos e drogas vegetais industrializados e oficializados pelo Ministério da Saúde no Brasil: regulamentação sanitária, abrangência e qualidade dos estudos pré-clínicos e clínicos. Doutorado em Saúde Pública, Escola Nacional de Saúde Pública Sergio Arouca, Fundação Oswaldo Cruz, Rio de Janeiro, Brasil.

Thiago, S. C. S., \& Tesser, C. D. (2011). Percepção de médicos e enfermeiros da Estratégia de Saúde da Família sobre terapias complementares. Revista de Saúde Pública, 45(2), 249-257.

Varela, D. S. S., \& Azevedo, D. M. (2014). Saberes e práticas fitoterápicas de médicos na estratégia saúde da família. Trabalho, Educação e Saúde, 12(2), $273-290$.

Vieira, S. C. H., Sólon, S., Vieira, M.C., \& Zárate, N. A. H. (2010). Levantamento de fitoterápicos manipulados em farmácias magistrais de Dourados-MS. Revista Brasileira de Farmacognosia, 20(1), 28-34. 